\title{
Are current clinical trials in diabetes addressing important issues in diabetes care?
}

\author{
W. C. Lakey • K. Barnard • B. C. Batch • \\ K. Chiswell • A. Tasneem • J. B. Green
}

Received: 19 November 2012 / Accepted: 26 February 2013 / Published online: 8 April 2013

(C) Springer-Verlag Berlin Heidelberg 2013

\begin{abstract}
Aims/hypothesis Clinical trials assessing interventions for treating and preventing diabetes mellitus and its complications are needed to inform evidence-based practice. To examine whether current studies adequately address these needs, we conducted a descriptive analysis of diabetesrelated trials registered with ClinicalTrials.gov from 2007 to 2010 .

Methods From a dataset including 96,346 studies registered in ClinicalTrials.gov downloaded on 27 September, 2010, a subset of 2,484 interventional trials was created by selecting trials with disease condition terms relevant to diabetes.

Results Of the diabetes-related trials, $74.8 \%$ had a primarily therapeutic purpose while $10 \%$ were preventive. Listed interventions included drugs $(63.1 \%)$ and behavioural $(11.7 \%)$. Most trials were designed to enrol $\leq 500(91.1 \%)$ or $\leq 100(58.6 \%)$ participants, with mean/median times to completion of $1.8 / 1.4$ years. Small percentages of trials targeted persons aged $\leq 18$ years $(3.7 \%)$ or $\geq 65$ years
\end{abstract}

Electronic supplementary material The online version of this article (doi:10.1007/s00125-013-2890-4) contains peer-reviewed but unedited supplementary material, which is available to authorised users.

W. C. Lakey · K. Barnard • B. C. Batch · J. B. Green $(\bowtie)$

Division of Endocrinology, Metabolism and Nutrition, Duke

University Medical Center, DUMC Box 3850, 2400 Pratt Street,

Room 7039, North Pavilion,

Durham, NC 27705, USA

e-mail: green094@mc.duke.edu

W. C. Lakey $\cdot$ K. Barnard • B. C. Batch $\cdot$ J. B. Green

Endocrinology Section, Durham Veterans Affairs Medical Center,

Durham, NC, USA

K. Barnard

Division of Endocrinology, Diabetes and Metabolism,

Stellenbosch University, Tygerberg, South Africa

K. Chiswell • A. Tasneem • J. B. Green

Duke Clinical Research Institute, Durham, NC, USA
(0.6\%), while $30.8 \%$ excluded patients $>65$ years and the majority excluded those $>75$ years. Funding sources included industry $(50.9 \%)$, NIH $(7.5 \%)$ or other, with most being single-centre trials of other sponsorship (37.7\%) or industryfunded multicentre studies (27.4\%). A small number of trials $(1.4 \%)$ listed primary outcomes including mortality or clinically significant cardiovascular complications. The distribution of trials by global region and US state does not correlate with prevalence of diabetes.

Conclusions/interpretation The majority of diabetes-related trials include small numbers of participants, exclude those at the extremes of age, are of short duration, involve drug therapy rather than preventive or non-drug interventions and do not focus upon significant cardiovascular outcomes. Recently registered diabetes trials may not sufficiently address important diabetes care issues or involve affected populations.

Keywords Clinical trials · Diabetes mellitus · Evidencebased medicine $\cdot$ Registry

\begin{tabular}{|c|c|}
\hline \multicolumn{2}{|c|}{ Abbreviations } \\
\hline EMA & European Medicines Agency \\
\hline FDA & US Food and Drug Administration \\
\hline IDF & International Diabetes Federation \\
\hline $\mathrm{MeSH}$ & Medical Subject Heading \\
\hline $\mathrm{NIH}$ & National Institutes of Health \\
\hline NLM & National Library of Medicine \\
\hline
\end{tabular}

\section{Introduction}

A total of 366 million people worldwide $-8.3 \%$ of the global population - have diabetes mellitus, and an additional 280 million persons have impaired glucose tolerance. Although the prevalence of diabetes varies greatly between 
ethnic groups and geographical regions, it disproportionately affects persons aged 65 years and older [1]. The public health toll of diabetes is on an upward trajectory, with its prevalence estimated to increase to more than 552 million persons worldwide by 2030 [1, 2]; furthermore, 2011 expenditures for diabetes and diabetes-related complications in the North American/Caribbean region alone are estimated at US\$223 billion [1]. Trials assessing interventions to prevent and treat diabetes and its complications are needed, but it is currently unclear whether the numerous clinical trials active in this therapeutic arena are capable of addressing deficiencies in our understanding of diabetes care.

ClinicalTrials.gov, a web-based registry maintained by the National Library of Medicine (NLM) at the US National Institutes of Health $(\mathrm{NIH})$, was created to give the public and healthcare providers easy access to information about clinical trials. In 2007, its scope was expanded to include the mandatory registration of all phase 2-4 interventional trials conducted under US regulatory auspices that have evaluated a drug, a biological therapy or a medical device [3]. Registration of trials with ClinicalTrials.gov or another comparable registry is also a prerequisite for publication in many peer-reviewed journals [4].

In order to evaluate the current state of clinical trials in diabetes, we conducted a descriptive analysis of diabetesrelated trials registered with ClinicalTrials.gov from 2007 to 2010 and compared this information with the current clinical picture of diabetes within the USA and worldwide to determine whether the current scope of trials permits us to effectively address disease prevention, management and safety of therapy in a diverse population. In particular, we examined whether recently registered trials were likely to enrol patients from high-risk or under-studied age groups, or from regions marked by a high disease prevalence. We also sought to define the percentage of trials focused on diabetes prevention as opposed to treatment, and the percentage of trials studying interventions other than drugs. Agencies including the European Medicines Agency (EMA) and the US Food and Drug Administration (FDA) have emphasised the need to better assess relative or comparative therapeutic effectiveness [5]; thus, we planned to evaluate the proportion of trials using active vs placebo comparators and the number of interventional arms in the trials. We also planned to describe the number of trials with outcomes that included clinically significant cardiovascular complications, and to identify trials focused upon areas of emerging interest, such as malignancies, bone metabolism/fractures or pancreatitis.

\section{Methods}

The methods used by ClinicalTrials.gov to register clinical trials have been described in detail elsewhere [6]. Briefly, trial sponsors and investigators from around the world can enter trial information through a web-based data entry system. The sample we examine in the present study includes trials registered to comply with statutory obligations, as well as those registered voluntarily to meet publication requirements or for other reasons.

Creation of the ClinicalTrials.gov dataset A dataset comprising 96,346 clinical studies registered in ClinicalTrials.gov was downloaded in XML format on 27 September 2010. This date of download was significant because it coincided with the enactment of the Food and Drug Administration Amendments Act 3 years prior to the date of the download and the corresponding legal obligation for sponsors to register applicable interventional trials. We next designed and implemented a relational database to facilitate the aggregate analysis of data from ClinicalTrials.gov, as described in detail elsewhere [7].

Creation of the diabetes study dataset Our analysis was restricted to studies categorised as being of 'interventional' study type that were registered with ClinicalTrials.gov from October 2007 to September 2010. The diabetes study dataset was created by using disease condition terms (both Medical Subject Heading [MeSH] and non-MeSH) provided by the trial data submitters, as well as additional $\mathrm{MeSH}$ condition terms generated by an NLM algorithm. A subset of the $2010 \mathrm{MeSH}$ thesaurus [8] and a list of non-MeSH disease condition terms provided by data submitters that appeared in at least five studies in the analysis dataset were reviewed and annotated by clinical specialists in endocrinology, metabolism and nutrition at Duke University Medical Center (W. C. Lakey, J. B. Green, B. C. Batch and K. Barnard) and the University of Oxford (M. A. Bethel).

As a first step, terms were annotated according to their relevance to the endocrinology domain. This domain is expansive and includes terms related to gland- and hormone-related diseases, as well as nutritional and metabolic conditions. Therefore, as a second step, terms selected for inclusion in the endocrinology domain were reviewed and annotated with respect to their relevance to diabetes and/or diabetes-related complications. In order to identify trials enrolling patients with prediabetes, terms such as 'impaired fasting glucose', 'impaired glucose tolerance' and 'hyperglycaemia' were included.

A total of 9,031 unique $\mathrm{MeSH}$ terms and 1,220 unique non-MeSH terms were reviewed. From this review, 1,031 unique MeSH terms and 146 unique non-MeSH terms were relevant to the endocrinology domain and used in the database search. A total of 8,302 studies were identified that had at least one condition term (MeSH or non-MeSH) relevant to endocrinology. In these studies, 1,353 unique $\mathrm{MeSH}$ terms occurred among the submitted conditions or NLM- 
generated MeSH terms, of which 19 were relevant to diabetes; of the 146 non-MeSH terms, 36 were relevant to diabetes (see electronic supplementary material [ESM] Table 1). Using the diabetes annotation, 2,484 studies were identified that had at least one condition term or condition $\mathrm{MeSH}$ term relevant to diabetes. Figure 1 displays a flow diagram showing the steps involved in creating the dataset.

Primary outcomes An investigator (J. B. Green) manually reviewed the listed primary trial outcomes because these data were entered as unstandardised free text. A review of all 2,500 free-text descriptions of primary outcomes was performed to identify outcomes of interest, including mortality or clinically significant cardiovascular complications such as myocardial infarction or stroke. Manual reviews were also performed for outcomes related to malignancies, bone metabolism/fractures or pancreatitis. This was followed by a text search for relevant keywords (J. B. Green, K. Chiswell and W. C. Lakey) to ensure that all listed outcomes of interest for the 2,484 diabetes studies had been identified (ESM Table 2).

Derived funding source The NLM defines the 'lead sponsor' for a trial as the organisation primarily responsible for study implementation and data analysis, and defines 'collaborators' as those who provide other meaningful trial-related support [9]. Agency names in these data elements are classified as 'industry', 'NIH', 'US federal (excluding NIH)' or 'other'. We derived the probable funding source from the 'lead sponsor' and 'collaborator' fields using the following algorithm: If the lead sponsor was from industry, or the NIH was neither a lead sponsor nor a collaborator and at least one collaborator was from industry, then the study was categorised as industry funded'. If the lead sponsor was not from industry, and the $\mathrm{NIH}$ was either a lead sponsor or a collaborator, then the study was categorised as 'NIH funded'. Otherwise, if the lead sponsor and collaborator fields were non-missing, the study was considered to be funded by 'other'.

Statistical methods Frequencies and percentages are provided for categorical trial characteristics. Unless otherwise indicated, missing values were excluded from the denominators before calculating the percentages. Means, medians and 25th and 75th percentiles are reported for continuous characteristics. For studies reporting an interventional model of 'single group' and the number of arms as ' 1 ', the value of allocation (if missing) was assigned as 'non-randomised' and the value of blinding (if missing) was assigned as 'open'.

\section{Results}

The overall characteristics of the diabetes-related trials are shown in Table 1. The 2,484 trials identified accounted for $6.0 \%$ of the 40,970 interventional trials overall and $6.4 \%$ of the 38,985 trials with a disease area classification. Of trials with an available start date, $81.2 \%$ began in 2007 or later. As of 27 September 2010, the largest proportions of diabetes trials were listed as 'completed' (36.3\%) or 'recruiting' (34.1\%), followed by 'active but not recruiting' (14.2\%) and 'not yet recruiting' (9\%). Of the trials with completion
Fig. 1 Flow diagram illustrating the creation of the diabetes study dataset

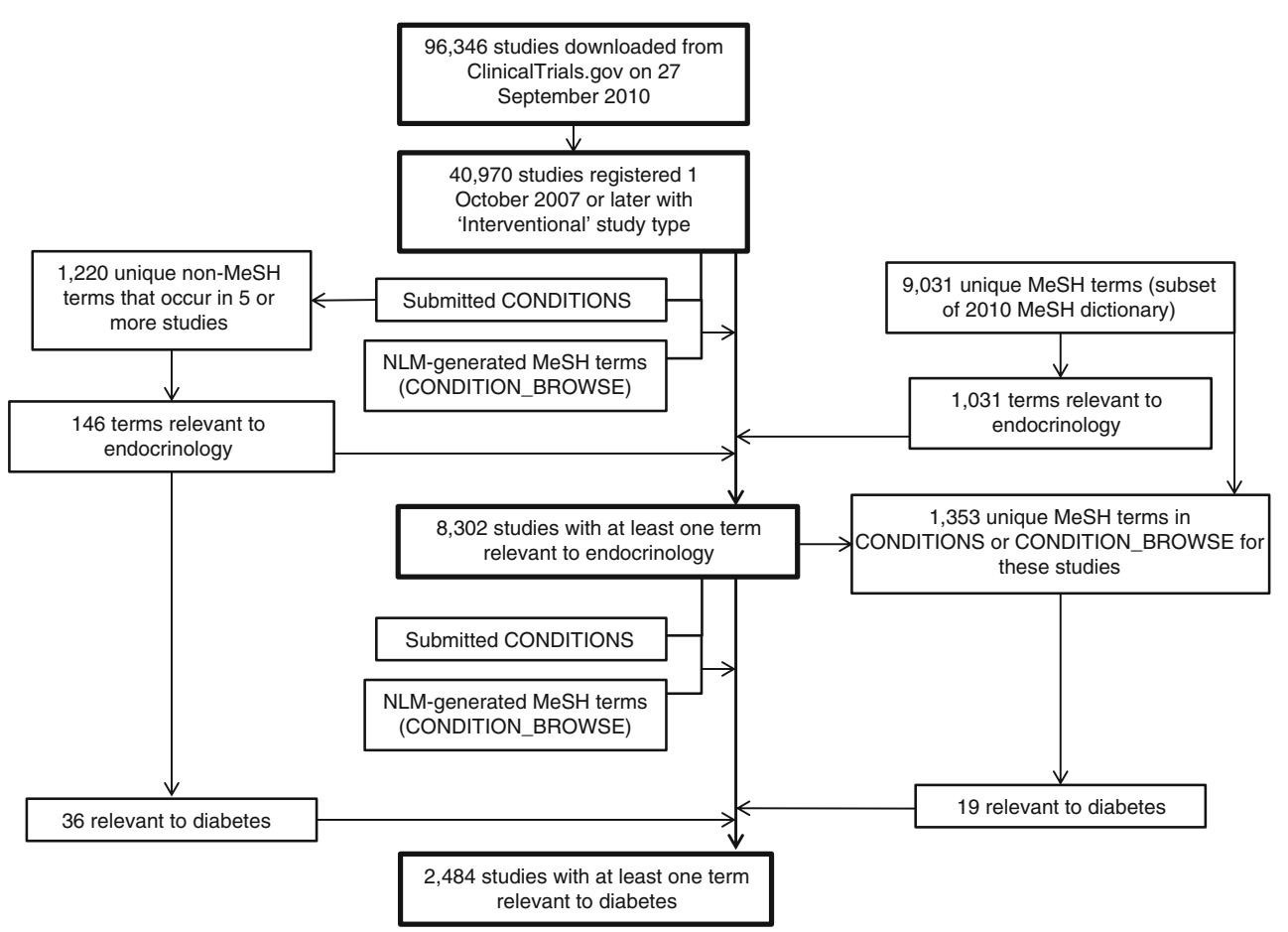


Table 1 Characteristics of diabetes-related interventional trials registered with ClinicalTrials.gov, 2007-2010

\begin{tabular}{ll}
\hline Characteristic & All diabetes studies \\
$N=2,484^{\mathrm{a}}$
\end{tabular}

Study start year, $n / N(\%)$
Before 2007
2007
2008
2009
2010 or later
Recruitment status as of 27 September $2010, n / N(\%)$
Not yet recruiting
Recruiting
Enrolling by invitation
Active, not recruiting
Completed
Suspended
Terminated
Withdrawn

$463 / 2,457(18.8)$
$279 / 2,457(11.4)$
$586 / 2,457(23.9)$
$652 / 2,457(26.5)$
$477 / 2,457(19.4)$ $846 / 2,484(34.1)$

$57 / 2,484(2.3)$

$353 / 2,484(14.2)$

$902 / 2,484(36.3)$

$3 / 2,484(0.1)$

$87 / 2,484(3.5)$

$13 / 2,484(0.5)$

Year follow-up for primary endpoint complete, $n / N(\%)$

Before 2007

$161 / 2,295(7.0)$

2007

2008

2009

2010

2011

2012 or later

Primary completion date type, $n / N(\%)$

Actual

Anticipated

Study duration ${ }^{\mathrm{b}}$

Mean \pm SD

Median (25th, 75th)

Min, max

Study phase, $n / N(\%)$

Phase 1

Phase 1 phase 2

Phase 2

Phase 2/phase 3

Phase 3

Phase 4

N/A

Enrolment, $n / N(\%)$

0

$1-10$

11-50

51-100

101-500

501-1,000

$>1,000$

227/2,295 (9.9)

453/2,295 (19.7)

$636 / 2,295$ (27.7)

436/2,295 (19.0)

295/2,295 (12.9)

$304 / 2,484(12.2)$

$84 / 2,484$ (3.4)

6/2,449 (0.2)

96/2,449 (3.9)

$840 / 2,449$ (34.3)

495/2,449 (20.2)

$793 / 2,449$ (32.4)

$152 / 2,449(6.2)$

$67 / 2,449(2.7)$
223/2,484 (9.0)

Table 1 (continued)

Characteristic

All diabetes studies $N=2,484^{\mathrm{a}}$

Enrolment type, $n / N(\%)$

Actual

$870 / 2,434$ (35.7)

Anticipated

$1,564 / 2,434(64.3)$

Primary purpose, $n / N(\%)$

Treatment

$1,740 / 2,327$ (74.8)

Prevention

233/2,327 (10.0)

Diagnostic

$54 / 2,327(2.3)$

Supportive care

$62 / 2,327(2.7)$

Screening

$5 / 2,327(0.2)$

Health services research $\quad 66 / 2,327(2.8)$

Basic science

$167 / 2,327(7.2)$

Study has one or more of these intervention types, $n / N(\%)^{\mathrm{c}}$

Drug

$1,568 / 2,484(63.1)$

Procedure

$130 / 2,484(5.2)$

$57 / 2,484(2.3)$

Biological/vaccine

Behavioural

290/2,484 (11.7)

Device

$186 / 2,484(7.5)$

2/2,484 (0.1)

$149 / 2,484(6.0)$

4/2,484 (0.2)

Radiation

Dietary supplement

Genetic

Other

290/2,484 (11.7)

Interventional model, $n / N(\%)$

Single group

$441 / 2,455(18.0)$

Parallel

Crossover

$1,597 / 2,455(65.1)$

$368 / 2,455(15.0)$

$49 / 2,455(2.0)$

887/2,293 (38.7) Factorial

$1,220 / 2,445$ (49.9)

$274 / 2,445(11.2)$

$951 / 2,445(38.9)$

$1.4(0.8,2.3) \quad$ Allocation, $n / N(\%)$

0.0, $12.1 \quad$ Randomised

$2,003 / 2,427(82.5)$

Non-randomised

$424 / 2,427(17.5)$

Number of arms, $n / N(\%)$

$1 / 2,351(0.0)$

0

$449 / 2,351(19.1)$

$78 / 2,484(3.1)$

$1,287 / 2,351(54.7)$

$314 / 2,351(13.4)$

$177 / 2,351(7.5)$

$123 / 2,351(5.2)$

Study has one or more of these arm types, $n / N(\%)^{\mathrm{d}}$
Experimental

Placebo comparator

Sham comparator

No intervention

Active comparator

Other
$1,389 / 1,891(73.5)$

$704 / 1,891(37.2)$

$22 / 1,891(1.2)$

$241 / 1,891(12.7)$

974/1,891 (51.5)

$87 / 1,891$ (4.6) 
Table 1 (continued)

\begin{tabular}{ll}
\hline Characteristic & All diabetes studies \\
$N=2,484^{\mathrm{a}}$
\end{tabular}

Sex, $n / N(\%)$
Female only
Male only
Both

Age, $n / N(\%)$

Maximum age $\leq 18$ years

Minimum age $\geq 18$ years

Excludes ages $>65$ years

Excludes ages $>75$ years

Minimum age $\geq 65$ years

Minimum age $\geq 75$ years

Number of primary outcomes

$n$

Mean \pm SD

Median (25th, 75th)

Min, max

Number of secondary outcomes

$n$

Mean \pm SD

Median (25th, 75th)

Min, max

Study classification, $n / N(\%)^{\mathrm{e}}$

Safety

Efficacy

Safety/efficacy

Bioequivalence

Bioavailability

Pharmacokinetics

Pharmacodynamics

Pharmacokinetics/pharmacodynamics

A primary outcome measures safety, $n / N(\%)$

A secondary outcome measures safety, $n / N(\%)$

Lead sponsor classification, $n / N(\%)$

Industry

$\mathrm{NIH}$

US federal

Other

$81 / 2,484(3.3)$

$118 / 2,484(4.8)$

$2,285 / 2,484(92.0)$

$92 / 2,484(3.7)$

$2,225 / 2,484(89.6)$

$764 / 2,484(30.8)$

$1,364 / 2,484(54.9)$

$15 / 2,484(0.6)$

$1 / 2,484(0.0)$

$1.2 \pm 0.80$

$1.0(1.0,1.0)$

1,16

2,440

$3.0 \pm 4.67$

$1.0(1.0,4.0)$

0,82

$122 / 2,032(6.0)$

$760 / 2,032(37.4)$

$927 / 2,032(45.6)$

$24 / 2,032(1.2)$

$10 / 2,032(0.5)$

$42 / 2,032(2.1)$

$54 / 2,032(2.7)$

93/2,032 (4.6)

432/2,321 (18.6)

$652 / 2,339(27.9)$

$1,062 / 2,484(42.8)$

$74 / 2,484(3.0)$

$37 / 2,484(1.5)$

$1,311 / 2,484(52.8)$

Lead sponsor or collaborator involvement, $n / N(\%)$ Industry lead or collaborator

NIH lead or collaborator

$1,276 / 2,484$ (51.4)

$189 / 2,484$ (7.6)

$62 / 2,484(2.5)$

US federal lead or collaborator

Other lead or collaborator

Funding source, $n / N(\%)^{\mathrm{f}}$

Industry

$\mathrm{NIH}$

Other

$1,383 / 2,484(55.7)$

$1,264 / 2,484(50.9)$

$187 / 2,484(7.5)$

$1,033 / 2,484(41.6)$

Funding/number of centres classification, $n / N(\%)$ Industry funded, single centre

493/2,237 (22.0)

Industry funded, multicentre
Table 1 (continued)

Characteristic

All diabetes studies $N=2,484^{\mathrm{a}}$

\begin{tabular}{lr}
\hline NIH funded, single centre & $137 / 2,237(6.1)$ \\
NIH funded, multicentre & $37 / 2,237(1.7)$ \\
Other, single centre & $843 / 2,237(37.7)$ \\
Other, multicentre & $115 / 2,237(5.1)$ \\
Number of facilities, $n / N(\%)$ & \\
Single facility & $1,473 / 2,237(65.8)$ \\
Multiple facilities & $764 / 2,237(34.2)$ \\
Number of facilities (for studies with multiple facilities)
\end{tabular}

$n$

Mean \pm SD

$34.6 \pm 60.25$

Median (25th, 75th)

$11.0(3.0,44.0)$

Min, max

2,741

Regions where studies have sites, $n / N(\%)^{\mathrm{g}}$

Africa

$78 / 2,237(3.5)$

Central America

$92 / 2,237(4.1)$

Eastern Asia

$303 / 2,237(13.5)$

Europe

$749 / 2,237(33.5)$

Middle East

$121 / 2,237(5.4)$

North America

$1,255 / 2,237(56.1)$

North Asia

$115 / 2,237(5.1)$

Pacifica

South America

$79 / 2,237(3.5)$

$156 / 2,237(7.0)$

South Asia

$142 / 2,237(6.3)$

South-East Asia

$83 / 2,237(3.7)$

Facility locations unknown

247/2,484 (9.9)

Has an enrolling facility in USA, $n / N(\%)$

$1,126 / 2,237(50.3)$

US/global location of facilities, $n / N(\%)$

USA only

907/2,237 (40.5)

Non-USA only

$1,111 / 2,237(49.7)$

Both USA and non-USA

$219 / 2,237(9.8)$

Oversight authority in USA

$1,332 / 2,484(53.6)$

${ }^{\text {a }}$ Missing values were excluded from the denominators before calculating the percentages

${ }^{\mathrm{b}}$ Derived: years from start date to completion of follow-up for primary endpoint

${ }^{\mathrm{c}}$ A study may have multiple interventions and could be counted in more than one category

${ }^{\mathrm{d}}$ Among 1,901 studies with $\geq 2$ arms. Ten studies are missing information on arm type. A study may have multiple arms and could be counted in more than one category

${ }^{\mathrm{e}}$ Indicates type of primary outcome or endpoint that the protocol is designed to evaluate. It is not a required field, and $18.2 \%$ of studies are missing this information

${ }^{\mathrm{f}}$ Derived from lead sponsor and collaborator fields using the derived funding source algorithm described under Methods

${ }^{\mathrm{g}}$ Studies may have facilities in multiple regions and be counted in more than one row. This information is only available for studies that have address information for facilities in the current study record. Region definitions can be found at www.clinicaltrials.gov/ct2/search/browse?brwse=locn_cat 
year data available, the majority were to have completed follow-up for their primary endpoint subsequent to 2008 and $12.9 \%$ were scheduled to complete follow-up in 2012 or later. The majority of these completion dates were anticipated $(61.3 \%)$ rather than actual $(38.7 \%)$. The mean and median times to primary trial completion (where available) were 1.8 and 1.4 years, respectively.

Diabetes trials were distributed relatively evenly across the early and late phases of development: $15.6 \%$ in phase 1 or phase $1 /$ phase $2,18.3 \%$ in phase 2 or phase $2 / 3,17.4 \%$ in phase 3 , and $16.9 \%$ in phase 4 . The largest percentage of trials overall (31.4\%) had study phase listed as 'not applicable'; however, smaller percentages of trials involving drug (13\%) or biological/vaccine interventions (14\%) did not identify a study phase. Among the 2, 449 trials listing enrolment, 91.1\% had an actual or anticipated enrolment of $\leq 500$ participants; $58.6 \%$ were designed to enrol $\leq 100$ participants.

Of the 2,327 trials with a primary purpose listed, the majority had a therapeutic purpose $(74.8 \%)$, followed by prevention (10\%) and basic science (7.2\%). Smaller percentages were focused upon diagnosis, supportive care, screening or health services research. Most trials involved drug interventions $(63.1 \%)$, followed by behavioural interventions $(11.7 \%)$, or had interventions classified as 'other'
$(11.7 \%)$. Smaller numbers included device-related $(7.5 \%)$ or procedural $(5.2 \%)$ interventions.

Of the trials for which data were available, $65.1 \%$ were parallel-design while $18 \%$ were described as single-arm; $49.9 \%$ were open-label and $38.9 \%$ were double-blind; and $82.5 \%$ of trials had a stated randomised allocation to therapy. Just over half (54.7\%) were described as having two treatment arms; $19.1 \%$ were single-arm and $13.4 \%$ had three arms. Disagreement in the percentages described as single-arm trials are related to the responses given to two separate questions. Among the trials reporting two or more study arms and with arm type available, $51.5 \%$ reported use of an active comparator arm while $37.2 \%$ reported the use of a placebo comparator arm. Trials of drug interventions were most likely to include two treatment arms (54.0\%), followed by one (15.8\%), three (14.7\%), four ( $8.73 \%$ ) or five or more $(6.8 \%)$ (data not shown).

Within the diabetes-related trials dataset, $92 \%$ accepted both male and female participants; much smaller percentages excluded women $(4.8 \%)$ or men $(3.3 \%)$. A total of $3.7 \%$ of trials limited enrolment to participants aged $\leq 18$ years, while $89.6 \%$ specifically excluded patients $<18$ years. Patients $>65$ years of age were excluded from $30.8 \%$ of studies; those $>75$ years were excluded from $54.9 \%$. Very few trials $(0.6 \%)$ selectively enrolled patients $\geq 65$ years; only one was designed to enrol
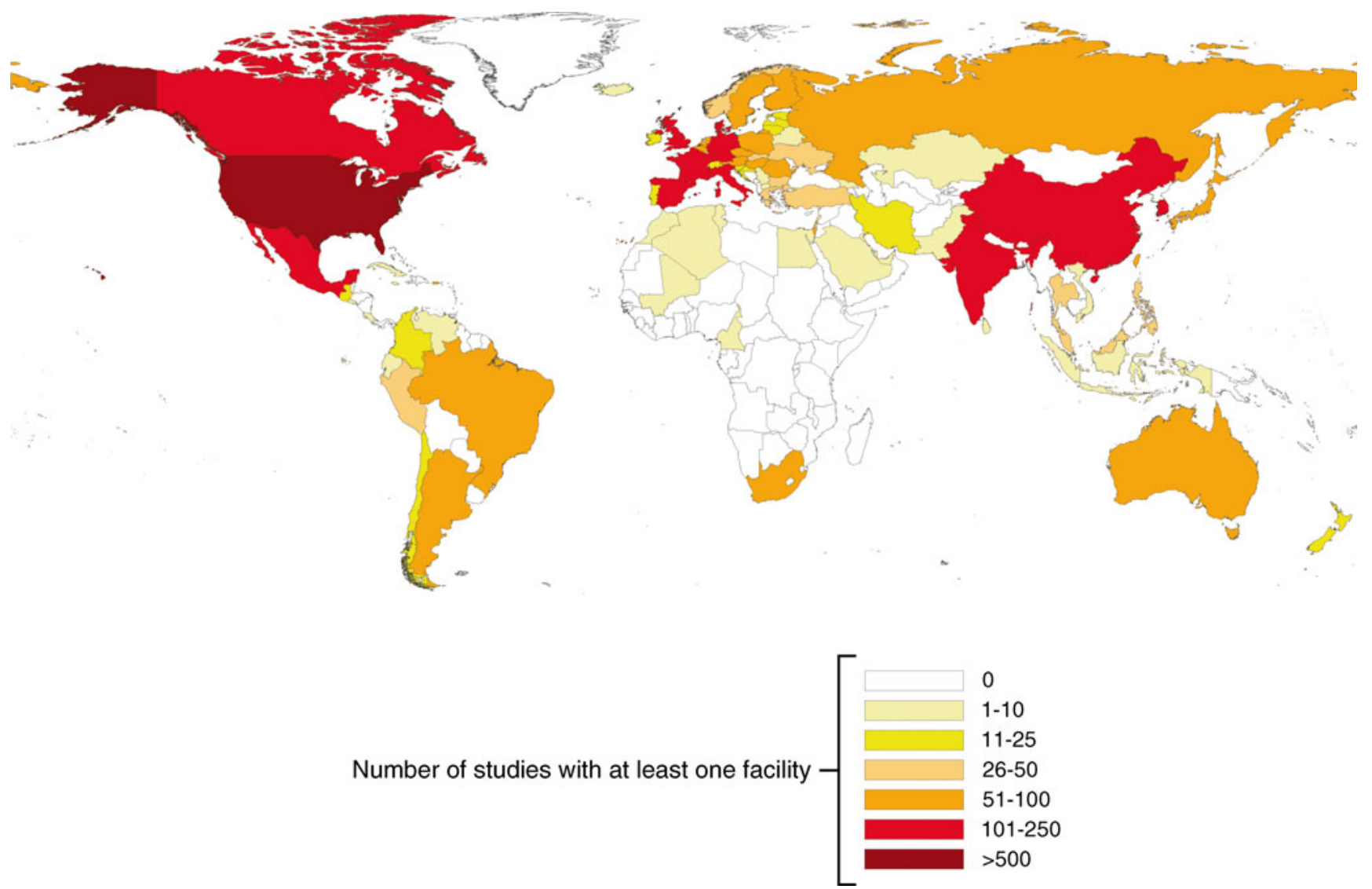

Fig. 2 Distribution of diabetes studies by country 
patients $\geq 75$ years. Data regarding planned or actual enrolment by race or ethnicity were not available.

A search for MeSH or non-MeSH terms specific to type 1 diabetes yielded 305 studies, or $12.3 \%$ of the total diabetes trials. However, this classification was not verified by manual review of all the trial descriptions, and thus may not accurately reflect the percentage of trials dedicated to study of this form of diabetes.

The mean and median numbers of listed trial primary outcome measures were 1.2 and 1, respectively. Among trials that listed secondary outcome measures, the mean and median numbers of secondary outcome measures were 3 and 1 , respectively. Of trials reporting study classification (the type of primary outcome that the protocol was designed to evaluate), the largest percentage had a safety/efficacy endpoint $(45.6 \%)$ and $37.4 \%$ had an efficacy endpoint. A total of $18.6 \%$ of studies had at least one primary outcome measuring safety, and $27.9 \%$ had at least one secondary safety outcome. A manual review of 2,500 free-text descriptions of trial outcomes yielded 35 studies $(1.4 \%)$ with at least one primary outcome related to mortality or clinically significant cardiovascular endpoints such as myocardial infarction or stroke, seven studies with at least one primary outcome related to bone metabolism, one study with a primary outcome related to malignancy, and none related to pancreatitis (ESM Table 2). One trial outcome description included both death and malignancy and was counted in both the 'cardiovascular' and 'malignancy' totals.

Lead sponsorship from industry was identified in $42.8 \%$ of trials; $51.4 \%$ had an industrial source as the lead sponsor or collaborator. Much smaller percentages were identified as having NIH $(3.0 \%)$ or U.S. federal lead sponsorship (1.5\%), although the NIH was identified as a lead sponsor or collaborator in $7.6 \%$ of trials. The largest percentage of lead sponsorship (52.8\%) was identified as being from other sources. Manual review indicated that many of these trials listed funding from universities or academic institutions. The derived funding source for the majority of the studies was industry (50.9\%), followed by other (41.6\%) and the NIH (7.5\%). Among the 2,237 trials for which data regarding sponsorship and number of centres were available, the largest percentage (37.7\%) comprised single-centre trials without funding from the NIH or industry. The next largest identifiable percentages were multicentre $(27.4 \%)$ or single-centre $(22.0 \%)$ trials with funding determined to be from industry. We found that the NIH provided funding for $6.1 \%$ of studies that were single-centre and $1.7 \%$ of studies that were multicentre trials.

Of the 2,237 studies that provided such information, 1,473 $(65.8 \%)$ took place at a single location. For studies with more than one location, the mean number of trial facilities was 34.6. Of the 764 multisite trials, $25 \%$ had two or three sites, $50 \%$ had $\leq 11$ sites and $75 \%$ had $\leq 44$ sites. The top $10 \%$ of multisite trials (73 trials in total) reported having more than 89 sites. A total of $40.5 \%$ of trials had facilities only in the USA, $49.7 \%$ were outside the US and $9.8 \%$ were conducted in the USA and other regions. The majority of studies $(56.1 \%)$ had at least one site located in North America, and 33.5\% had at least one site in Europe. Much smaller percentages of studies were located in Asian regions (Eastern Asia, 13.5\%; South Asia, 6.3\%; North Asia, 5.1\%; South-East Asia, 3.7\%), South or Central America (7\% and $4.1 \%$, respectively), the Middle East (5.4\%), Africa $(3.5 \%)$ or Pacifica $(3.5 \%)$. The distribution of trials by country is shown in Fig. 2.

\section{Discussion}

A review of the data available from diabetes-related trials registered in ClinicalTrials.gov from 2007 to 2010 provides an important window on the current clinical research enterprise in this therapeutic area. Our descriptive analysis found that the majority of registered trials involve drug therapies rather than preventive or non-drug interventions. Trials appear to include relatively small numbers of patients, are primarily conducted at single sites and are of fairly short duration. Trials often exclude children and elderly participants, their global distribution does not correlate with regional disease prevalence, and only small numbers of trials have focused upon mortality or clinically significant cardiovascular complications.

The International Diabetes Federation (IDF) and the ADA emphasise diabetes prevention as a focus of future research $[10,11]$. Previous trials have demonstrated that various lifestyle and pharmacological interventions may delay the onset of diabetes in high-risk persons [12-15]; however, additional study is needed to enhance the implementation of preventive strategies in practice and assess the utility of novel interventions. And despite convincing evidence that intensive glycaemic control minimises the onset and progression of complications [16, 17], a significant percentage of persons with diabetes have not achieved optimal glycaemic control $[18,19]$. Further study regarding the translation of effective educational, preventive and therapeutic interventions in the community setting is also encouraged by the ADA [11]. We have found that most diabetesrelated studies in ClinicalTrials.gov focus on treatment (usually drug-related rather than behavioural), while only small percentages are primarily concerned with prevention, health services research, supportive care, diagnosis or screening. And although the ideal proportion of trials focused on prevention has not been established, the current trials portfolio, comprising studies with smaller sample sizes and shorter durations, appears to be inadequate for expanding and refining preventive efforts or translating effective care strategies into the community setting.

The IDF, ADA and others have emphasised the need for trials designed to compare the effects of therapies in diverse, 
high-risk and representative populations $[10,11,20]$. The prevalence of diabetes varies by global region and country [1] and by race/ethnicity [21]. In addition, rates of complications including diabetic retinopathy, lower extremity amputation and end-stage renal disease vary among ethnic groups [22-25]. To achieve the greatest impact upon clinical care, trials should enrol patients representative of populations disproportionately affected by diabetes, including high-risk patients and those $\geq 65$ years. A better understanding of responses to interventions among diverse individuals and groups may inform individualised treatments of greater effectiveness and tolerability [26, 27].

Race and ethnicity of trial populations are not required fields for registering studies with ClinicalTrials.gov; therefore, there is no readily available information for this category within the dataset. However, the location of trials within countries provides insight into the relationship between clinical trial activities and highly affected populations. Registration with ClinicalTrials.gov is not required for studies taking place outside US jurisdiction; nevertheless, approximately half of the trials registered in ClinicalTrials.gov did not have any US sites. Thus, although our dataset affords an incomplete view of trials activity worldwide, it still likely that it provides a reasonably accurate global perspective.

The IDF list of the ten locations most affected by diabetes includes multiple Middle Eastern countries in which the prevalence of diabetes among adults is approximately $20 \%$ [1]. However, our analysis suggests that this region is minimally involved in diabetes-related trials. A comparison of trial activities in countries with the highest prevalence of diabetes among adults reveals over $500(1,126)$ trials in the USA; however, we also noted state- and regional-level exceptions to this, as detailed in the ESM text and ESM Fig. 1. China, India and Mexico participated in 101-250 trials each; however, the Russian Federation (12.6 million persons affected) and Brazil (12.4 million affected) are involved in only 51-100 registered trials despite heavy disease burdens [1].

Trials registered in ClinicalTrials.gov are predominantly conducted in North America, Western Europe and a small number of countries in Asia. Notably, most of Africa is either uninvolved or minimally involved in registered studies. Thus, current trials appear unlikely to provide significant insight into the management of patients from many highly affected or under-studied areas.

The ClinicalTrials.gov database permits a review of the ages of participants sought for (or excluded from) trials. Although those aged 40-59 years constitute the largest number of persons affected by diabetes worldwide, older persons are at greatest risk of the disease. For example, $26.9 \%$ of US residents $\geq 65$ years were estimated to have diabetes in 2010 [20]. Our analysis found that persons $>65$ years were excluded from $30.8 \%$ of trials, and that the majority of trials excluded those aged $>75$ years. Thus, the current clinical research portfolio may not allow us to robustly address issues in older persons with diabetes.

Less than $4 \%$ of registered trials targeted the enrolment of participants $\leq 18$ years. This may be appropriate given the number of children affected by diabetes; however, the estimated 3\% annual increase in incidence of type 1 diabetes may warrant greater representation [1]. Furthermore, the increase in type 2 diabetes among adolescents, particularly noticeable in wealthier nations, is of considerable concern. It is unclear whether findings obtained from adults with diabetes are readily translatable to paediatric/adolescent populations. The inclusion of younger participants in diabetes trials is essential to ensure safe and effective clinical interventions for these groups, particularly given their risk of developing disease complications early in life. Current clinical trials do not appear to be appropriately positioned to address issues related to disease prevention or management in the young.

Organisations including the US Institute of Medicine have encouraged comparative effectiveness trials to comprehensively assess the benefits and risks of multiple therapeutic options for diabetes and other diseases [27, 28]. Trials comparing the safety, effectiveness and durability of the many glucose-lowering therapies now available will create a reliable evidence base for clinical care guidelines.

The majority of currently registered diabetes trials have a parallel intervention model; however, most have two treatment arms $(54.7 \%)$ or one treatment arm (19.1\%), leaving only a small percentage with three or more arms. Among trials with two or more arms, $51.5 \%$ include an active comparator. Furthermore, the relatively short duration typical of these trials may compromise our ability to ascertain the durability of therapeutic interventions or the effects of interventions upon longterm complications. The single-site nature and limited enrolment of most trials are likely to limit the conclusions drawn from their results. Therefore, the current set of trials may not contribute to meaningful changes in recommendations for care.

Diabetes care organisations worldwide have emphasised a need to minimise diabetes-related complications. Groups including the ADA have strategically prioritised investigations that will enhance our understanding of these complications, including cardiovascular disease [11]. The relationship between glycaemic therapeutic targets, hypoglycaemia and cardiovascular complications remains inadequately understood and contentious, despite multiple recent outcomes studies [29-32]. In addition, scrutiny of the cardiovascular effects of individual glucose-lowering therapies has increased following concerns about rosiglitazone and other drugs in development [33], resulting in new FDA and EMA guidelines for evaluating the cardiovascular safety of new glucose-lowering agents [34, $35]$. Of the 2,439 trials in the dataset with available outcomes descriptions listed, only 35 show a primary outcome related to mortality or clinically significant cardiovascular endpoints (e.g. myocardial infarction or stroke). Only small numbers of 
trials reported primary outcomes related to bone metabolism, malignancy or pancreatitis despite significant clinical interest in the relationship between these issues and glucose-lowering therapy or diabetes itself [36-39].

There are limitations to our ability to draw firm conclusions from the data available, many of which have been previously described [40]. Although ClinicalTrials.gov encompasses a substantial proportion of clinical trials and an estimated $80 \%$ of studies in the WHO portal, it does not include all studies performed worldwide [40]. Incorporating non-duplicate trials registered with other international databases would have provided a more complete global perspective; however, such an undertaking would require relatively intensive curation efforts to ensure that duplicate studies were removed and categories appropriately matched, and thus lies beyond the scope of the present work.

Requirements and methods for collecting information about trials have changed over time, and data completeness and quality are variable - an unsurprising finding, as the data collection was not initially designed to support aggregate analysis. Missing data, classification of data as 'other' in many circumstances, and non-standardised free-text descriptions also complicated our analysis, particularly when reviewing data related to funding sources and trial outcomes. Funding sources are also classified in a manner most relevant to US-based trials. We were able to identify the presence of a trial within countries and specific US states; however, the number of unique sites per country or state could not be determined, thus limiting our capacity to assess the proportion of trial activity in relation to the population density of interest within a given area. In addition, information about facilities that had not yet been activated when the database was downloaded or had been removed from the current study record is excluded from this investigation. Future refinements to data collection may permit a more complete and sophisticated analysis of trials characteristics.

With respect to the data analysis, the non-hierarchical MeSH classifications may categorise a condition in multiple locations, potentially leading to false positives upon querying for a specific condition. Endocrinology experts at two institutions annotated the database; however, this annotation has not yet been externally validated. In this initial overview, we did not examine whether various characteristics have changed over time and are thus unable to discern meaningful trends in the design or implementation of clinical trials.

In summary, this descriptive analysis of data from the ClinicalTrials.gov registry provides a broad overview of interventional clinical studies related to diabetes. Although many trials will provide valuable information upon completion, our review suggests that the current portfolio does not adequately address disease prevention, management or therapeutic safety. This information may be meaningful in the allocation of future research activities and resources.
Acknowledgements The authors wish to thank M. A. Bethel (Deputy Director of the Diabetes Trials Unit, Oxford Centre for Diabetes, Endocrinology and Metabolism, University of Oxford, Oxford, UK) for her assistance in selecting terms for inclusion in the endocrinology domain. The authors also thank J. McCall (employee of the Duke Clinical Research Institute, Durham, NC, USA), for editorial assistance in preparing this manuscript; he received no compensation for this work other than his usual salary. Part of these research findings were delivered via oral presentation at the EASD Scientific Sessions in October of 2012.

Funding Financial support for this work was provided by grant U19FD003800 from the FDA, awarded to Duke University for the Clinical Trials Transformation Initiative.

Duality of interest The authors declare that there is no duality of interest associated with this manuscript.

Contribution statement All authors made substantial contributions to the conception and design of the analysis, and the acquisition and analysis of the data. All authors also either drafted or critically revised this manuscript for important intellectual content and approved the final version to be published.

\section{References}

1. International Diabetes Federation (2011) IDF Diabetes Atlas, 5th edn. International Diabetes Federation, Brussels. Available from www.idf.org/diabetesatlas, accessed 3 October 2012

2. World Health Organization. Country and regional data on diabetes. Available from www.who.int/diabetes/facts/world_figures/en/ index $3 . h t m l$, accessed 3 October 2012

3. US Food and Drug Administration. Public Law 110-85. The Food and Drug Administration Amendments Act of 2007. Available from www.fda.gov/RegulatoryInformation/Legislation/ FederalFoodDrugandCosmeticActFDCAct/Significant AmendmentstotheFDCAct/FoodandDrugAdministration AmendmentsActof2007/FullTextofFDAAALaw/default.htm, accessed 4 October 2012

4. DeAngelis CD, Drazen JM, Frizelle FA et al (2004) Clinical trial registration: a statement from the International Committee of Medical Journal Editors. JAMA 292:1363-1364

5. European Medicines Agency. Road Map to 2015. Available from www.ema.europa.eu/ema/index.jsp?curl=pages/about_us/general/ general_content_000292.jsp\&mid=WC0b01ac05800293a4, accessed 16 November 2012

6. ClinicalTrials.gov Protocol Registration System. PRS information. Available from http://prsinfo.ClinicalTrials.gov, accessed 4 October 2012

7. Tasneem A, Aberle L, Ananth H et al (2012) The database for aggregate analysis of ClinicalTrials.gov (AACT) and subsequent regrouping by clinical specialty. PLoS One 7:e33677

8. US National Library of Medicine (2010) Medical Subject Headings thesaurus (2010). Available from www.nlm.nih.gov/mesh/ 2010/introduction/introduction.html, accessed 4 April 2012

9. ClinicalTrials.gov Protocol Data Element Definitions (Draft). Available from http://prsinfo.ClinicalTrials.gov/definitions.html, accessed 4 October 2012

10. Alberti K, Zimmet P, Shaw J (2007) International Diabetes Federation: a consensus on type 2 diabetes prevention. Diabet Med $24: 451-463$ 
11. Fonseca V, Kirkman M, Darsow T, Ratner R (2012) The American Diabetes Association diabetes research perspective. Diabetes Care 35:1380-1387

12. Diabetes Prevention Program Research Group (2002) Reduction in the incidence of type 2 diabetes mellitus with lifestyle intervention or metformin. N Engl J Med 346:393-403

13. NAVIGATOR Study Group (2010) Effect of valsartan on the incidence of diabetes and cardiovascular events. N Engl J Med 362:1477-1490

14. Chiasson JL, Josse RG, Gomis R et al (2002) Acarbose for prevention of type 2 diabetes mellitus: the STOP-NIDDM Randomized Trial. Lancet 359:2072-2077

15. DREAM (Diabetes Reduction Assessment with Ramipril and Rosiglitazone Medication) Trial Investigators (2006) Effect of rosiglitazone on the frequency of diabetes in patients with impaired glucose tolerance or impaired fasting glucose: a randomized controlled trial. Lancet 368:1096-1105

16. Diabetes Control and Complications Trial Research Group (1993) The effect of intensive treatment of diabetes on the development and progression of long-term complications in insulin-dependent diabetes mellitus. N Engl J Med 329:977-986

17. UK Prospective Diabetes Study (UKPDS) Group (1998) Intensive blood-glucose control with sulphonylureas or insulin compared with conventional treatment and risk of complications in patients with type 2 diabetes (UKPDS 33). Lancet 352:837-853

18. Ong KL, Cheung BM, Wong LY et al (2008) Prevalence, treatment, and control of diagnosed diabetes in the US National Health and Nutrition Examination Survey 1999-2004. Ann Epidemiol $18: 222-229$

19. Saaddine JB, Cadwell B, Gregg EW et al (2006) Improvements in diabetes processes of care and intermediate outcomes: Unites States, 1998-2002. Ann Intern Med 144:467-474

20. International Diabetes Federation Bridges. Bringing Research in Diabetes to Global Environments and Systems. Available from www.idf.org/bridges/general-information, accessed 17 January 2013

21. Centers for Disease Control and Prevention. National diabetes fact sheet: national estimates and general information on diabetes and prediabetes in the Unites States, 2011. US Department of Health and Human Services, Centers for Disease Control and Prevention, Atlanta, GA, 2011

22. Zhang X, Saaddine JB, Chou CF et al (2010) Prevalence of diabetic retinopathy in the United States, 2005-2008. JAMA 304:649-656

23. Wong TY, Klein R, Islam FM et al (2006) Diabetic retinopathy in a multi-ethnic cohort in the United States. Am J Ophthalmol 141:446-455

24. Resnick HE, Valsania P, Phillips CL (1999) Diabetes mellitus and nontraumatic lower extremity amputation in black and white Americans: the National Health and Nutrition Examination Survey Epidemiologic Follow-up Study, 1971-1992. Arch Intern Med 159:2470-2475

25. Karter AJ, Ferrara A, Liu JY et al (2002) Ethnic disparities in diabetic complications in an insured population. JAMA 287:2519-2527
26. Smith RJ, Nathan DM, Arslanian SA, Groop L, Rizza RA, Rotter JI (2010) Individualizing therapies in type 2 diabetes mellitus based on patient characteristics: what we know and what we need to know. J Clin Endocrinol Metabol 95:15661574

27. Collins F. Comparative effectiveness and personalized medicine: an essential interface (conference), 19-21 October, 2010, National Institutes of Health, Bethesda, MD. Conference video available from http://videocast.nih.gov/Summary.asp?file=16209, accessed 4 October 2012

28. National Research Council (2009) Initial national priorities for comparative effectiveness research. National Academies Press, Washington, DC

29. Gerstein HC, Miller ME, Byington RP et al (2008) Effects of intensive glucose lowering in type 2 diabetes. $\mathrm{N}$ Engl $\mathrm{J}$ Med 358:2545-2559

30. Duckworth W, Abraira C, Moritz T et al (2009) Glucose control and vascular complications in veterans with type 2 diabetes. N Engl J Med 360:129-139

31. Patel A, MacMahon S, Chalmers J et al (2008) Intensive blood glucose control and vascular outcomes in patients with type 2 diabetes. N Engl J Med 358:2560-2572

32. Holman R, Paul S, Bethel A et al (2008) 10-year follow-up of intensive glucose control in type 2 diabetes. N Engl J Med 359:1577-1589

33. Nissen SE, Wolski K (2007) Effect of rosiglitazone on the risk of myocardial infarction and death from cardiovascular causes. N Engl J Med 356:2457-2471

34. US Food and Drug Administration (2008) Guidance for industry: diabetes mellitus - evaluating cardiovascular risk in new antidiabetic therapies to treat type 2 diabetes. Available from www.fda.gov/ downloads/Drugs/GuidanceComplianceRegulatoryInformation/ Guidances/ucm071627.pdf, accessed 4 October 2012

35. European Medicines Agency (2010) Guideline on clinical investigation of medicinal products in the treatment of diabetes mellitus. Available from www.emea.europa.eu/docs/en_GB/document library/Scientific guideline/2010/02/WC500073570.pdf, accessed 4 October 2012

36. Smith U, Gale EA (2009) Does diabetes therapy influence the risk of cancer? Diabetologia 52:1699-1708

37. Currie CJ, Poole CD, Gale EA (2009) The influence of glucoselowering therapies on cancer risk in type 2 diabetes. Diabetologia 52:1766-1777

38. Meier C, Kraenzlin M, Bodmer M et al (2008) Use of thiazolidinediones and fracture risk. Arch Intern Med 168:820 825

39. Drucker DJ, Sherman SI, Bergenstal RM, Buse JB (2011) The safety of incretin-based therapies - review of the scientific evidence. J Clin Endocrinol Metab 96:2027-2031

40. Califf R, Zarin D, Kramer J et al (2012) Characteristics of clinical trials registered in ClinicalTrials.gov, 2007-2010. JAMA 307:1838-1847 